\title{
CIUDADANÍA Y AUTONOMÍA EN TRABAJO SOCIAL: EL PAPEL DE LOS SABERES POLÍTICOS Y CIENTÍFICO TÉCNICOS
}

\author{
MARÍA CRISTINA MELANO \\ Universidad de Buenos Aires (Argentina)
}

\section{RESUMEN}

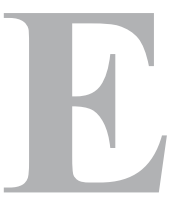

1 artículo desarrolla la temática de identidad profesional del Trabajo Social a partir de abordar los conceptos de tradición, historia, sentido, experiencia. Señala la presencia de la heterogeneidad en dicha construcción. Incorpora la categoría "identidad de frontera", clave para comprender esta profesión. Relaciona la identidad con la autonomía profesional y su incidencia en la construcción y defensa de ciudadanía. Expone el concepto de disciplina y en ese marco destaca el papel de los saberes técnicos para el campo profesional.

Palabras clave: identidad, identidad de frontera, autonomía, disciplina, saberes técnicos.

ABSTRACT: Identity/citizenship and autonomy in social work: the role of technical political and scientific knowledge

This article examines the subject of professional identity within Social Work, approaching the issue from the concepts of tradition, history, sense and experience. The existence of heterogeneity in this construction is identified, and the category of "borderline identity", key to understanding the profession, is incorporated. Furthermore, identity is related to professional autonomy and the impact of this on the construction and defence of citizenship. The concept of discipline is presented and, within this framework, the role of technical knowledge in the profession is highlighted.

Key words: identity, borderline identity, autonomy, discipline, technical knowledge.

Facultad de Ciencias Sociales. Universidad de Buenos Aires (Argentina). 


\section{INTRODUCCION}

Podríamos hipotetizar que una mayor autonomía del Trabajo Social puede tributar a la ampliación y a la calidad de la ciudadanía.

Pero..., ¿en qué consiste la autonomía? ¿Y qué relación guarda con la identidad?

Y, si disponemos de autonomía o de cuotas parte de autonomía..., ¿desde la/s misma/s pueden reforzarse procesos de ciudadanización?

¿Qué papel juegan saberes en dicha construcción?

¿Qué importancia le asigna actualmente el Trabajo Social a dichos saberes?

En este discurso, incluiremos miradas filosóficas: en tal sentido, destacamos que la Filosofía nos aporta ojos extranjeros, de extrañamiento, miradas laterales para captar cuestiones y nos da sostén en la tarea de designar. Compartimos el planteo heideggeriano de que "la ciencia no piensa" pues quien piensa es la Filosofía, aún cuando por sí sola no produce verdades.

Asimismo, abrevaremos en la Epistemología para abordar la cuestión de los saberes, pues esa ciencia contribuye a abrir caminos de percepción y favorece desarrollos heurísticos. Adherimos a una concepción epistemológica crítica que sustenta la responsabilidad moral y el reconocimiento de los rasgos epocales en la reflexión sobre el conocimiento científico, articulado con la historia.

Apelaremos a conceptos de las diferentes ciencias sociales (Sociología, Ciencias Políticas, Antropología, Derecho) y Ciencias de la Educación, entre otras.

Este ensayo procura problematizar algunas "verdades" para hacer aparecer la "aletheia" e instalar cuestiones opacadas actualmente en el mundo académico y en el mundo del trabajo.

\section{IDENTIDAD Y AUTONOMIA}

\subsection{Algunas claves para pensar el concepto de identidad}

Hablar de autonomía del Trabajo Social remite a un tema quizás remanido que ha ocupado un lugar significativo en el escenario del debate profesional en la última década: el de la identidad de la profesión.

Resulta difícil ceder ante la tentación de abordar esta cuestión, que hemos tratado en un trabajo escrito hace muchos años (motivado por la confrontación

1 HEIDEGGER, Martin, La ciencia no piensa”, en: La Nave (periódico de psicología, filosofía y literatura), Buenos Aires, Vol. 3, No 16, 1997, pp. 8-9; traducción del equipo de la Fundación Centro Psicoanalítico Argentino.

2 Entendida como velos que al ser corridos revelan aquello que está por detrás. 
ante algunas posiciones circulantes que cristalizaban el perfil profesional ${ }^{3}$ ) y que trataremos de ampliar en este texto.

El término latino identidad (identitas derivado de ídem, lo mismo) designa a lo que es idéntico a sí mismo y que se diferencia de la otredad, a un ser que se reconoce a sí mismo como diferente de algo exterior, sin desconocer los propios cambios. Y esa esencia, en el caso del Trabajo Social, puede descubrirse si analizamos su tradición, su historia, su sentido, su telos o los fines a los que tiende. También sus prácticas, temas a los que aludiremos, de modo transversal, más adelante.

Estas palabras claves que intentaremos definir, nos arrojan algunas pistas para pensarnos.

La tradición (del latín traditio, de tradere, hacer pasar a otro, transmitir) ocupa un lugar central en el moldeado de identidades grupales ${ }^{4}$. Lo que traemos y transmitimos es aquello a lo que asignamos importancia: ideas, consideraciones éticas, supuestos, principios, prácticas, técnicas. Nos son transmitidas en los procesos formativos (a través de circuitos educativos formales, no formales, informales) o durante el desempeño de nuestras prácticas profesionales. Apelamos a la tradición para vivir el presente y pensar el futuro de la profesión.

Hans G. Gadamer, señalaba que los presupuestos o pre-juicios (en el sentido etimológico de juicios previos) constituyen una memoria cultural ${ }^{5}$.

Validando a Heidegger, afirmaba: (...) 'Son los prejuicios no percibidos los que con su dominio nos vuelven sordos hacia la cosa de que nos habla la tradición. El razonamiento de Heidegger, hace comprensible esta tradición porque descubre las premisas ontológicas del concepto de subjetividad”.

Heidegger «asegura» el tema científico introduciéndolo y poniéndolo en juego en la comprensión de la tradición. En esto consiste la concreción de la conciencia histórica de la que se trata en el comprender. Sólo este reconocimiento del carácter esencialmente prejuicioso de toda comprensión confiere al problema hermenéutico toda la agudeza de su dimensión ${ }^{6}$.

Gadamer nos indica que la tradición no es sólo memoria, sino que confiere sentidos y, a partir de ellos, es posible abrir nuevas cartografías.

Pero, además, la tradición de la profesión no está sólo presente en nosotros, trabajadores sociales, también lo está en el imaginario social y en el de las personas que acuden a demandar nuestros servicios.

3 Véase MELANO, M.C.: el capítulo Identidad Profesional - La insoportable levedad de las fronterizaciones" en: Un Trabajo Social para los nuevos tiempos. La construcción de la ciudadanía. Editorial Hvmanitas. Buenos Aires. 2001 o Revista de Trabajo Social No 64-.Escuela de Trabajo Social de la Pontificia Universidad Católica de Chile. 1994

4 SAltalamachiA, H.: Historia de vida. Ediciones CiJUP. Puerto Rico. 1992. El autor, aludiendo a las sociedades tradicionales, refiere a que "los antiguos guerreros de la vida participaban en la lenta, pero indispensable tarea, de ir moldeando las identidades grupales".

5 GADAMER, H.: Verdad y método, Ed. Sígueme, Salamanca 1977, p.336.

6 Ibídem .pág. 337. 
La historia, como desarrolla Edward Carr, "es un proceso y no se puede aislar un fragmento del proceso y estudiarlo independientemente (...) todo está interconectado" ". El pasado no necesariamente es retrógrado: Umberto Eco advierte de que, en los tiempos actuales, hipnotizados por la velocidad, nuestras sociedades, en muchas cuestiones, queriendo avanzar, retroceden. Tampoco creemos que se debe entronizar el pasado; no siempre "todo tiempo pasado fue mejor". Lo que no podemos desconocer es la incidencia del ayer en la construcción de las identidades individuales y colectivas presentes y futuras: desde lo que fuimos somos y seremos.

La historia refleja nuestra posiciones en el tiempo: comprender el pasado es comprender el presente, que se relaciona con errores, secretos, relatos.

La trayectoria de la profesión se construye en torno a nuestras prácticas y discursos. Conocerla y comprenderla nos permite dialogar con referentes de las cuestiones que nos interesa aprehender, resignificar el lugar de los actores en la hechura de los procesos sociales.

La historia es acontecer, res gesta, da cuenta de procesos que se dan en un contexto y que son construidos por actores portadores de subjetividades. Y es rerun gestarum, es relato y como tal siempre interpretado. La interpretación de esta historia reciente está atravesada de tensiones y las tensiones identitarias se hacen presentes en la historia ${ }^{8}$.

\subsection{El sentido}

Alude a lo subjetivo, a las razones manifiestas, o aquellas latentes, asociadas con la idea de razón de ser, de dirección. Lo real es real reconstruido. Por lo expuesto, la cuestión del sentido es difícil de capturar a través de la investigación empírica, si bien hechos y datos pueden permitir captarlo... "Esto no quiere decir que deba tratárselo de manera torpe o ilógica, sino que hay razones que la razón no alcanza a comprender".

El sentido procura captar la conciencia de sí y se logra también a través de la percepción de los cambios o las modificaciones de perspectivas, tengan éstos causación interna o externa. Está histórica y culturalmente condicionado y, por ende, nuevos contextos pueden abrir nuevos sentidos o posibilitar su fuga o ausencia. De ahí, la necesidad de pensar el sentido como multiplicidad abierta (y también abierta a las disyunciones)10. Todo hacer humano, profesional o no, produce no sólo productos, servicios, arte. También construye subjetividad y relatos en torno a su mismidad.

7 CARR, E.: ¿Qué es la historia? Ed. Ariel. Barcelona 2003. Pág. 43.

8 En el caso de Argentina, el Trabajo Social se autodefinió como agente de cambio, concientizador, mediatizador, agente externo, entre otros. En nuestro país, el Trabajo Social no nace antimoderno, es fundacionalmente moderno, si bien se hipotetiza que sus primeros egresados asimilaban la profesión con apostolado.

9 ROZITCHNER, A.: El sentido no es una ciencia. Diario La Nación-Buenos Aires, 19 de octubre de 2005.

10 Véase DIAZ, E.: Entre la tecnociencia y el deseo. Editorial Biblos. Buenos Aires 2007. Pág. 65. La autora define disyunción como incompatibilidad, como lo contrario a la complementariedad, como alternativas no componibles, como los términos de una disyunción : o una u otra, persistiendo ambas 


\subsection{Trabajo Social ¿Identidad/es de frontera?}

Como substrato de estas cuestiones, se hallan presentes otras tensiones identitarias que simplemente enunciaremos, tales como debatirse entre:

- el ser y el deseo de ser, para asegurar su reconocimiento,

- el ser y el deber ser,

- la lealtad institucional o abogacía de los usuarios,

- la persistencia y reproducción de los modelos socio-político-económicos vs. confrontación,

- burocratización vs. iniciativa-innovación, desarrollo de la dimensión instituyente,

- control social vs. vigencia, consolidación, ampliación de los derechos humanos y ciudadanos,

- expertez vs. politicidad,

- procesos vs. resultados y

- ciencia vs. tecnología/técnica.

Estos temas problematizados y problematizables, sobre los cuales giramos permanentemente, nos sugieren la inclusión de una nueva categoría para caracterizar nuestra identidad, la de identidad de frontera. Pues no es de desconsiderar que trabajamos en los ámbitos donde las condiciones de pobreza, (material, pero también política, simbólica, educativa), el problema social o el dolor humano están presentes y, por el otro lado, somos indiscutiblemente profesionales, profesionales en el límite.

Y esta condición de profesionales amerita una especial consideración en la conformación de esta identidad de frontera. Nos debe alertar: tenemos que ser conscientes de nuestra posición de pequeños burgueses, presente aún cuando provengamos de sectores más desfavorecidos. François Vatin se interroga acerca de si [El artista, el pensador...o el escritor de antes (y nos permitimos agregar los profesionales del trabajo social) ¿son los oenicos que pueden encontrar sentido y placer en su trabajo? $]^{11} \mathrm{Y}$ alerta sobre la tendencia de éstos a "mirar el trabajo de las masas a través del prisma de sus privilegios" en torno a lo cual se pregunta "Y sin embargo, ¿no hay ahi un soberano desprecio?"

En la misma línea, cuando nos titulamos, a veces ostentando los títulos, cual aristócrata exhibe sus blasones nobiliarios, certificamos nuestros conocimientos de grado o postgrado y, a través de ellos, accedemos al ejercicio legítimo de la profesión, obtenemos una autoridad social legitimada en torno a nuestras competencias y saberes.

Compartimos que hay profesión "cuando un tipo de actividad no se ejerce más que mediante la adquisición de una formación controlada, la sumisión a reglas y normas de conducta entre los miembros y respecto a los no miembros

11 VATIN, F.: Ensayos de sociología y epistemología del trabajo. Lumen-Humanitas, Buenos Aires, 2004 o en http://www.ceil-piette.gov.ar/docpub/libros/trcs.html. 
y la adhesión de una técnica» ${ }^{12}$ y nos preguntamos con Wilemsky, si la profesionalización no sería más que una manera por la cual los grupos sociales intentarían explotar y monopolizar ciertos recursos apropiados a ciertas necesidades sociales ${ }^{13}$, es decir, la monopolización de atribuciones de poder. La titulación nos confiere cuotas partes de poder. Desde nuestra base profesional ocupamos posiciones, desempeñamos cargos en distintos niveles de intervención, realizamos funciones, implementamos actividades, ejercemos poder.

Nietzsche, filósofo provocativo, cuyo pensamiento ha sido fuente de los análisis desarrollados por múltiples corrientes, señalaba que, desde oscuras relaciones de poder, se inventaron la poesía, la religión ${ }^{14}$. Nos permitimos agregar también las profesiones, entre ellas la nuestra, acerca de cuya posible identidad de frontera nos interrogamos.

Si acordamos en que la frontera representa un corte, que expresa un límite, pero que ineludiblemente invita a ser traspasado, compartiremos que en nuestro proceso de profesionalización hemos ultra-pasado las fronteras de la microactuación y las de la acción meramente asistencial. Y, desde esa identidad de frontera, atendemos problemas sociales diversos, en disímiles campos, desde diferentes niveles (micro-meso-macro) y unidades de intervención. Impulsamos objetivos específicos acordes a las misiones y funciones "declamadas" por las instituciones efectoras de política social en las que nos insertamos laboralmente, desde perspectivas no siempre homogéneas. Resalta como una verdad de Perogrullo que nuestro quehacer no se caracteriza por la homogeneidad.

$\mathrm{Si}$, como señalaba Nietzsche "todo concepto, surge del afirmar como igual lo no igual"15, el de identidad no escapa a esta consideración. Quizá podamos encontrar alguna pista para elucidar la cuestión si, en vez de pensar la cuestión de identidad como homogeneidad, pensamos en el sentido, por una parte como conocimiento al que podemos darle valor y, por otra, como significado de nuestras prácticas.

Pues la identidad profesional alude a ese ser particular, el Trabajo Social, con multiplicidad de significados que guardan un principio de identidad, un centro unificador, un ousias para los griegos, una sustancia con diferentes substancias, una esencia. Nos unifica un telos, pautas de conducta, concepciones de legitimidad, la enunciación de valores, la posición de bisagra entre el hoy y el mañana de las poblaciones con las que trabajamos, un rol de posible condicionante de futuro que puede ser alterativo o reproductor de su presente.

También los acervos teórico, metodológico, estratégico, táctico e instrumental construidos históricamente son indicadores de la pertenencia de los profesionales al Trabajo Social. Desde esta identidad heterogénea, ¿qué autonomía es susceptible de ser portada por el Trabajo Social?

12 AM. Carr y A. Wilson (1933), en G. Thines y A. Lemperner. Diccionario general de ciencias humanas, Ediciones Cátedra, Madrid 1978 p. 727).

13 Ibídem.

14 Citado por Foucalt, M.: La verdad y las formas jurídicas. Gedisa. Barcelona 1980. Pág. 21.

15 Nietzsche, F., Sobre verdad y mentira en sentido extramoral en Discurso y Realidad. San Miguel de Tucumán. 1987. Universidad de Tucumán. pág. 75. 
La preocupación por la autonomía está presente ya en Mary Richmond, cuando enuncia, al definir el trabajo social de casos individuales: "me limito a las formas del Servicio Social que reúnen las tres siguientes condiciones: primeramente ser practicadas por personas competentes, en segundo lugar, ocuparse de casos dificiles que necesitan una intervención prolongada e intensiva $y$ en tercer lugar ser realizada con una relativa independencia y sin restricciones arbitrarias $"{ }^{\prime 16}$. Amerita destacar la agudeza de apreciación de quien fue la impulsora de nuestra profesión y dedicó sus esfuerzos a argumentar sobre la necesidad de delimitarla como campo específico.

El concepto de autos (sí mismo) nomos (ley) refiere a quien vive según su propia ley o se gobierna por su propia ley. Epistemológicamente, alude a la heterogeneidad e independencia de las disciplinas o profesiones para recortar objetos de estudio y seleccionar y definir métodos de adquisición de conocimientos y /o de intervención. Desde el plano ético, la autonomía supone tener libertad para darse su propia ley moral, pero esta libertad se enmarca en un contexto socio-cultural y político que le fija techos, por ello la autonomía de cualquier profesión, entre ellas la nuestra, es intrínsecamente relativa...

De modo genérico, podemos identificar algunos avances en materia de autonomía del campo, asociados a su proceso de profesionalización: contamos con leyes profesionales, consejos profesionales -libremente conformados-, códigos de ética -auto-promulgados- que aplican sanciones a través de nuestros tribunales disciplinarios. Nuestras carreras tienen membresía universitaria, delimitan sus propios planes de estudio, enmarcados en las normativas educativas vigentes ${ }^{17}$. Pero, aproximarnos a la verdad, en nuestro caso, en términos de conocer la autonomía del Trabajo Social, supone también analizar, como señala Foucault, cómo se transforman los dominios de saber en prácticas sociales ${ }^{18}$ y cómo son las relaciones de lucha y de poder en el campo profesional. Foucault también nos expresa que Nietzsche ha destacado que "fue (...) de pequeñez en pequeñez que finalmente se formaron las grandes cosas "19.

Podemos inferir de ello la relevancia que conlleva recuperar, analizar e investigar las intervenciones profesionales cotidianas, desde las de nivel micro a las de nivel macro, los discursos en torno a las mismas, establecer zonas de cruce entre rigurosas auto-evaluaciones y hetero-evaluaciones para identificar el nivel y la calidad de nuestra autonomía. Consideramos que estamos en deuda con estos análisis: nuestros datos estadísticos son frágiles y es débil nuestra presencia en el escenario público dando cuenta de nuestra gestión, así como fijando posicionamientos en torno a las cuestiones objeto de nuestra actuación.

16 Richmond, M.: El Caso Social Individual. Ed. Hvmanitas. Bs. As. 1977. Pág. 61 a 62. (subrayado nuestro).

17 Hemos desarrollado estas cuestiones en Desprenderse para devenir. Travesías y destinos del Trabajo Social Argentino. En "La profesionalización en Trabajo Social. Rupturas y continuidades, de la Reconceptualización a la construcción de proyectos ético- políticos". Capítulo IV. Coordinadora Margarita Rozas Pagaza. Espacio Editorial. Buenos Aires 2007. Pág. 33 a 39.

18 Foucault, M. La verdad y las formas jurídicas. Gedisa. Barcelona 1980. Pág. 13,14.

Foucault se caracterizaba a sí mismo como un filósofo de "bajo vuelo", aludiendo a la condición de tener los pies en la tierra y buscar referentes empíricos a cuestiones filosóficas, en muchos casos planteadas provocativamente por Nietzsche), de ahí este planteo que desarrolla en el texto mencionado.

19 Citado por Foucault, M.: La verdad y las formas jurídicas. Gedisa. Barcelona 1980. Pág. 21. 
¿Resulta suficiente discursear sobre posicionamientos ético-políticos que se conservan endogámicamente si estos discursos no se distribuyen y circulan en la sociedad, si no hay voluntad política para que den lugar a prácticas políticas? ¿Alcanza con demandar o proclamar la autonomía del campo si no la operacionalizamos con acciones?

Nuestra autonomía depende de la legitimidad de origen del Trabajo Social, de nuestras competencias formativas, de la legitimidad institucional que nos es conferida por vía contractual, pero también entre otras condiciones de la legitimidad social alcanzada a través de nuestro ejercicio, asociada a nuestra capacidad para poner temas en la agenda pública y proponer alternativas de atención/resolución, y se vincula con nuestro prestigio nuestra solvencia argumentativa, la productividad de los contenidos de nuestro discurso, nuestro acervo teórico, metodológico, técnico, táctico, nuestra destreza técnica, nuestra habilidad para entramar interacciones, para establecer alianzas estratégicas, para hacer política.

Pero, así como nuestra identidad no es homogénea, nuestra influencia y, en consecuencia, nuestra autonomía, como profesión, tampoco lo es, porque los otros (usuarios-profesionales-expertos-políticos) constituyen la "otredad" que también se inserta en luchas de poder simbólicas. Cuanto mayor sea la autonomía del Trabajo Social, mayor posibilidad tendrá de direccionarse hacia criterios de libertad, igualdad, justicia, justicia social.

En tal sentido, los trabajadores sociales tenemos competencias para proveer escucha, conocemos dispositivos legales institucionales y organizacionales para atender problemas sociales. Y disponemos de información susceptible de transferir a los ciudadanos acerca de a quién/es puede/n apelar para el cumplimiento efectivo de sus derechos: cómo incrementar sus posibilidades de elección para asumir responsabilidades en la toma de decisiones públicas, cómo ejercer el contralor de la transparencia de los actos de gobierno, cómo protegerse ante los abusos de los poderes económicos y políticos.

No obstante, y más allá de la autonomía de la que disponemos, estas informaciones suelen "brillar por su ausencia", cuando desplegamos nuestras prácticas interventivas. Si disponemos de autonomía, podemos trabajar en pos de profundizar la calidad de la participación, vinculada ésta al intercambio de las ideas, las posiciones, las diferencias entre las distintas esferas de la vida pública, a fin de arribar a consensos que vayan más allá de la negociación entre grupos de poder o de la representación política que proviene del mandato de las urnas. Podemos contribuir a que los ciudadanos identifiquen oportunidades, efectúen aprendizajes abiertos, que aporten a la definición y priorización de problemas, al diseño, ejecución, monitoreo de políticas públicas, al control del presupuesto destinado a la política social.

El nivel de profesionalización alcanzado por el Trabajo Social hace posible pensar que podemos accionar en el sentido de ciudadanización. En el caso argentino, durante el último trienio, hemos incrementado nuestra injerencia en el diseño de políticas públicas y, paralelamente, la credibilidad de las poblaciones y usuarios con relación a la práctica profesional se ha visto fortalecida. Valga recordar que la política es una dimensión de la vida social, a través de la cual 
se impulsan acciones que promueven la instauración de nuevos escenarios. Ampliar la ciudadanía también aumenta la legitimidad del Estado.

Y, para contribuir a la construcción y consolidación de la ciudadanía, las disciplinas requieren de diferentes saberes. Foucault señala que una disciplina se define por un ámbito de objetos, un conjunto de métodos, un corpus de proposiciones consideradas verdaderas, un juego de reglas y de definiciones, de técnicas y de instrumentos ${ }^{20}$. En ese conjunto de proposiciones consideradas verdaderas, pueden ser incluidos los saberes temáticos (teóricos o populares), el conocimiento del marco normativo existente, los saberes del ámbito estadístico, así como los saberes de los que los ciudadanos son portadores, que son centrales para un pertinente desempeño de la profesión del Trabajo Social ${ }^{21}$.

\subsection{Los saberes técnicos}

Focalizaremos en la cuestión de los saberes técnicos por entender que reviste interés en las ciencias aplicadas, atento a que los objetos de intervención no sólo se transforman según la forma de mirarlos, sino también a partir de la realización de operaciones concretas a través de las cuales son aplicados teorías y principios más generales. Los saberes técnicos y las operaciones procedimentales para investigar o actuar constituyen una cuestión central de las disciplinas científicas: han sido y son objeto significativo de estudio en las ciencias sociales (Derecho, Sociología, entre otros) atento a que su utilización errónea puede viciar de nulidad las hipótesis que pretenden probarse en las prácticas científicas o profesionales...

La impericia en el accionar de una profesión -que, entre otras cuestiones, puede vincularse a la inadecuada aplicación de instrumentos o procedimientos- es una de las causales de mala praxis, de ahí la importancia que se le asigna a esta temática en el marco de las profesiones con basamento científico. A diferencia, los trabajadores sociales que encuadramos nuestras prácticas en el marco de las ciencias sociales, en la última década le hemos asignado a las cuestiones operativas escasa atención ${ }^{22}$.

Parecería que la consideración de la técnica como expresión del proyecto del capitalismo moderno en cuanto a racionalidad instrumental ha incidido en la escasa atención que se presta actualmente al saber técnico en el ámbito académico. Asimismo, las cuestiones técnicas también han quedado relegadas en las prácticas de campo (en los ámbitos territoriales o institucionales), los profesionales usan la técnica pero parecerían limitarla al plano del hacer, a una operatoria efectuada desde la habilidad, producto de la experiencia, sin que sea atravesada por los procesos de reflexión que una intervención fundada demanda.

20 Foucault, M. El orden del discurso. Barcelona. Tusket. 1980.

21 Véase Melano, M.C.: "Trabajo social: sujeto y ciudadanía” en Compartiendo Notas. El trabajo social en la contemporaneidad. UNLa. Remedios de Escalada.

22 Hemos señalado en numerosas publicaciones que detallamos en la bibliografia la escasa consideración que se dispensa en nuestro medio a la reflexión sobre saberes técnicos. 
Pero debemos acordar que el progreso humano se implica en transformaciones técnicas y no pueden contrarrestarse los efectos (negativos) del capitalismo sin el uso de la técnica.

Gilbert Simondon, quien re-introdujo la cuestión de la técnica en los debates acerca de la crisis del trabajo, intentando instalar la vocación productiva y no simplemente económica del mismo, destacó la importancia de reducir -si no romper- la alienación del trabajo y señaló la necesidad de recuperar el sentido de la técnica: "Para reducir la alienación, habría que devolver a la unidad de la actividad técnica el aspecto de trabajo. (...) de aplicación concreta que implica el uso del cuerpo, y la interacción de los funcionamientos; el trabajo debe volverse actividad técnica" ${ }^{23}$.

La presencia de nuevas subjetividades, de sujetos no esperados por la sociedad ni por las instituciones efectoras de políticas públicas, lleva a re-plantearnos nuestras formas de intervención asociadas éstas con contextualizaciones témporo-espaciales, con conceptualizaciones y sistemas teóricos que requieren ser reformulados. Nos marcan nuevas preguntas, sugieren nuevas inscripciones sociales y noveles formas de acogida de la población y sus problemas. Los cambios epocales y las características de principios del milenio sugieren la importancia de poner el foco en las circunstancias en que se producen las intervenciones y los obstáculos que entorpecen su despliegue, de seleccionar alternativas adecuadas a situaciones concretas, no estereotipadas, que contemplen la cultura, los recursos y las potencialidades de los sujetos que participan en los procesos de intervención profesional... En un escenario social que demanda innovación, las cuestiones técnicas tienen una presencia substantiva.

Porque el saber técnico va más allá del respeto a prescripciones procedimentales: el rechazo a iniciativas e innovaciones es obstáculo a todo avance productivo. También los avances de las técnicas se vinculan, además de a los mencionados sistemas teóricos y conceptualizaciones, a contextos de validez y a propuestas metodológicas en ámbitos socio-económico-políticos.

Los instrumentos dan cuenta de los modos de ver y de ser en el mundo, del sentido de las intervenciones, constituyen su polea de transmisión, favorecen la estructuración de procesos. Problematizarlos implica sistematizar procedimientos, reflexionar en torno a su naturaleza, que opaca e invisiviliza el uso del poder que puede ejercerse a través de su utilización. De ahí la necesidad de que, como profesionales del Trabajo Social, analicemos qué estrategias y tácticas, estilos y modos de discurso se vinculan con la eficacia y la eficiencia de las intervenciones.

Si deseamos que el Trabajo Social sea disciplina, deberíamos interrogarnos acerca de si no debemos asignarle a la técnica el papel que inviste, analizarla desde lo epistemológico, fundamentarla, validarla científica y prácticamente. Esta última forma de validar, impone pensar en sus fundamentos, en las fuentes en que se abreva para su construcción, en los criterios de diseño y aplicación, en qué poder nos confiere y cómo construimos poder a través de las mismas.

23 Simondon, G.: Du mode d'existence des objets techniques, París, Aubier-Montaigne, 1969, p. 241p. 251-252. 
La técnica tiene un poder que se encubre, es medio para investigar e intervenir, para aportar pruebas acerca de la validez o no de un curso de acción. $\mathrm{Su}$ desarrollo es cuestión central si creemos que podemos intervenir modificando situaciones liminares que afectan a nuestras sociedades, en pos de la libertad, de la justicia, de la igualdad, de la intensidad de la vida de los ciudadanos.

Por nuestra parte, creemos que es tiempo de dejar el lamento y cambiarlo por la "ciencia gaya", por la alegría de intervenir, porque tenemos un sentido y luchamos por causas nobles, porque creemos que el Trabajo Social puede contribuir al goce del aquí ahora y del tiempo por venir. Y ello no es viable si obviamos las cuestiones técnicas.

\section{BIBLIOGRAFÍA}

BACHELARD, G.: Epistemología (textos escogidos por Lecourt, D.), Anagrama, Barcelona 1973.

CARR, A.M. y. WILSON, A. (1933), en Thines, G. y Lemperner, A.: Diccionario general de ciencias humanas, Ediciones Cátedra, Madrid 1978.

CARR, E. ¿Qué es la historia? Ed. Ariel. Barcelona 2003

DELEUZE, G. Conversaciones. Editorial Pre Textos. Valencia 1999.

FOUCAULT, M. La verdad y las formas jurídicas. Gedisa. Barcelona 1980.

FOUCAULT, M. El orden del discurso. Barcelona. Tusket. 1980.

FOURIEZ, G.: Alfabetización cientifica y tecnológica. Bruselas De Boeck 1994.

GADAMER, H.: Verdad y método, Ed. Sígueme, Salamanca 1977.

GLADU, É.: La contribution de Gilbert Simondon à l'étude de la technique. Étudiante en maîtrise de communication. Université de Montréal. 2000.

HEIDEGGER, M.: "La ciencia no piensa", en: La Nave (periódico de psicología, filosofía y literatura), Buenos Aires, Vol. 3, No 16, 1997, pp. 8-9; traducción del equipo de la Fundación Centro Psicoanalítico Argentino.

MELANO, María Cristina “Aventuras y desventuras de los saberes técnicos”. Revista Trabajo Social N ${ }^{a}$ 5. Departamento de Trabajo Social. Universidad de Antioquia. Medellín. Colombia. 2007. pág. 35 a 54

MELANO, M.C.: "Desprenderse para devenir. Travesías y destinos del Trabajo Social Argentino". En La profesionalización en Trabajo Social. Rupturas y continuidades, de la Reconceptualización a la construcción de proyectos ético- politicos. Capitulo IV. Coordinadora Margarita Rozas Pagaza. Espacio Editorial. Buenos Aires 2007. Pág. 33 a 39.

MELANO, M.C.: "El valor de la palabra y los instrumentos de intervención del Trabajo Social". Revista Servicios Sociales y Política Social. No78. Pág. 67 a 77.Consejo General de Colegios Oficiales de Diplomados en Trabajo Social. Madrid.

MELANO, M.C.: "Identidad Profesional. La insoportable levedad de las fronterizaciones" en: Un Trabajo Social para los nuevos tiempos. La construcción de la ciudadanía. Editorial Hvmanitas. Buenos Aires. 2001 o Revista de Trabajo Social No 64-.Escuela de Trabajo Social de la Pontificia Universidad Católica de Chile. 1994.

MELANO, M.C.: "La travesía de la intervención. Teoría, método y técnicas participativas en trabajo social”. Revista Servicios Sociales y Politica Social. № 58. Madrid. 2002. 
MELANO, M.C.: "Técnicas Dramáticas y Procesos Socio Educativos". Revista Trabajo Social No 18. Montevideo- Uruguay -Bs. As. 2000.

MELANO, M.C.: “Técnicas Participativas. Entre el arte y la ciencia» en Revista Cuadernos de Trabajo Social. Carrera de Trabajo Social. Facultad de Ciencias Sociales UBA-AÑO 1- N 1-Buenos Aires, noviembre de 1995.

MELANO, M.C.: “Trabajo social: sujeto y ciudadanía” en Compartiendo Notas. El trabajo social en la contemporaneidad. UNLa. Remedios de Escalada .

NIETZSCHE, F.: "Sobre verdad y mentira en sentido extramoral" en Discurso y Realidad. San Miguel de Tucumán. 1987.

ROZITCHNER, A.: El sentido no es una ciencia. Diario La Nación. Buenos Aires, 19 de octubre de 2005.

SALTALAMACHIA, H.: Historia de vida. Ediciones CIJUP. Puerto Rico. 1992.

STEINER, G.: Los tres desafios de la humanidad. Diario La Nación. Suplemento Cultura. Domingo 3 de setiembre de 2006.

VATIN, F.: Trabajo, ciencias y sociedad. Ensayos de sociología y epistemología del trabajo. Lumen-Humanitas, Buenos Aires, 2004 o en http://www.ceil-piette.gov.ar/docpub/libros/trcs. html

www.scielo.br: Intersecções entre o ambiente e a realidade técnica: contribuições do pensamento de Simondon, G. .Ambiente\& sociedade.Print ISSN 1414-753X. 